\title{
Simulação e análise do comportamento da onda Eletromagnética de dados GPR no monitoramento dos postos de abastecimento
}

\author{
Silva,D.A,UFAM; Tejada,Z.R.P,UFAM; PIEDADE,A.A, UFPA
}

Copyright 2019, SBGf - Sociedade Brasileira de Geofísica

This paper was prepared for presentation during the $16^{\text {th }}$ International Congress of the Brazilian Geophysical Society held in Rio de Janeiro, Brazil, 19-22 August 2019.

Contents of this paper were reviewed by the Technical Committee of the $16^{\text {th }}$ International Congress of the Brazilian Geophysical Society and do not necessarily represent any position of the SBG, its oflicers or members. Electronic reproduction of the Brazilian Geophysical Society is prohibited.

\section{Resumo}

A variety of environmental problems occur due to the leakage of hydrocarbons. Applied geophysics methodologies to environmental studies show great results in the investigation of contaminating. GPR is a practical and fast geophysical method in such investigations, because the electromagnetic signal assists in the delimitation of the contaminating, addressing direct techniques of investigation like boreholes and wells. This study, three environmental situations were modeled at a filling station, before the leakage of the underground tank, after the leakage, and when this was remedied, then, the simulation was done perpendicular to the direction of the aquifer flow using two different antennas (100MHz and $250 \mathrm{MHz})$. It was possible to evaluate the more resistive zones identified as shade zones. The results show that GPR signal after the leakage is much attenuated in the contamination phases, after the remediation the behavior of the EM wave tends to recover great part (existence of new minerals) the original properties of the soil. This fact could be result of a bacteriological activity in the residue or increase of dissolved salts in the soil caused by acid $\mathrm{pH}$ of polluting.

\section{Introdução}

Segundo a ANM (Agência Nacional do Petróleo), até o começo de dezembro do ano de 2016, o número de postos de gasolina na cidade de Manaus era de 284. Atualmente, este valor possivelmente sofreu um acréscimo. E, segundo Lima (2010), a vida útil dos tanques de armazenamento de combustível dos postos da cidade está próxima do fim, aumentando dessa forma, a possibilidade de ocorrência de vazamento ou derramamento de gasolina. Ainda segundo - mesmo autor, riscos carcinogênicos pela exposição ao benzeno foram encontrados mais de $60 \%$ na cidade Manaus. Desta forma, é necessário que haja maior monitoramento tanto das regiões afetadas pela presença de benzeno quanto aquelas ainda livres da contaminação, visando a prevenção. Para isso, se faz necessário o incentivo ao estudo e pesquisas na área para que haja desenvolvimento e aperfeiçoamento de métodos que identifiquem zonas contaminadas e que impeçam novos derramamentos.

A contaminação do solo é preocupante porque pode atingir o lençol freático, e tornar a água imprópria para o consumo. Os vazamentos em postos de combustíveis provocam grandes problemas ao meio ambiente, (Prommer et al. (1999)) e os resíduos tóxicos podem penetrar no solo e atingir altas profundidades e tamanhos não estimáveis, considerado-se este como um passivo ambiental. A série de compostos denominados BTEX, compreende o benzeno, tolueno, etilbenzeno e xilenos. Estes efluentes apresentam um elevado potencial de poluição, representando um sério risco ao meio ambiente e ao ser humano (Mello (2007)). São vários os motivos pelo qual o vazamento pode existir: seja a pressão, material do tanque, armazenamento dos líquidos inflamáveis, manutenção dos equipamentos, instalações inadequadas e o monitoramento de entrada e saída do combustível, assim como o ambiente geológico e as forças tensionais aos quais os tanques estão submetidos. Entretanto, o fato mais frequente é que muitos tanques tem mais de 25 anos de uso e dessa forma,a possibilidade de ocorrerem vazamentos é extremamente grande, principalmente pelo surgimento de rachaduras ou corrosão (Tiburtius et al. (2004)).

Ante tal problemática, os métodos geofísicos, como o Ground Penetrating Radar (GPR) mostraram bons resultados na identificação de locais contaminados por "líquidos imiscíveis em fase não aquosa" - NAPL's (Dehaini (2001), Penner (2005) ; de Castro and Branco (2003)) e verificaram que a amplitude do sinal eletromagnético (EM) é menor na parte superior da pluma de hidrocarboneto, podendo ser provocada pela fase residual do mesmo. Autores como Moreira and Dourado (2007), identificam essas áreas como zonas de sombra. O objetivo principal deste trabalho foi de avaliar o comportamento da onda eletromagnética de um posto de combustível em diferentes ambientes (antes da contaminação, durante a contaminação e após a remediação) e assim avaliar a aplicabilidade do método ante situações de monitoramento.

Este estudo realiza uma análise comparativa entre radargramas obtidos antes de acontecer o vazamento do tanque subterrâneo, posterior ao vazamento e quando este foi remediado, bem como a simulação dos ambientes mencionados. Dessa forma será possível avaliar a influência dos compostos nas propriedades físicas do solo, alterando a condutividade elétrica nas diferentes fases de contaminação e assim, analisar a resposta nos seções proporcionadas pelo GPR. Por fim, o realce do refletor após a remediação segundo o estudo, mostra como o refletor é visivelmente perceptível à ausência de compostos. 


\section{Metodologia}

Dentro da estrutura de trabalho, o principal alvo na modelagem é analisar o comportamento da onda eletromagnética diante de possíveis vazamentos de hidrocarbonetos, em particular a gasolina, visto que é constituída por hidrocarbonetos mais leves e apresenta maior solubilidade, maior volatilidade, menor viscosidade, além de ter maior mobilidade no solo, tendendo a ser o hidrocarboneto com maior potencial de impacto ambiental.

Para isso, organizou-se o estudo da siguinte forma:

- Analisar o comportamento do hidrocarboneto na zona vadosa e zona saturada.

- Modelagem de três situações diferentes em GPR: Antes do meio ser contaminado, durante a contaminação e por último, quando este contaminante foi tratado ao longo dos anos.

- Simulação das três modelagens.

- Por fim, analisar o comportamento da onda dos dados sintético e compará-los aos dados reais de Dehaini (2001).

\section{Comportamento do hidrocarboneto na Zona vadosa e saturada}

O processo inicial de contaminação de águas subterrâneas por constituintes de gasolina (benzeno, tolueno, etilbenzeno e xileno "BTEX") ocorre a partir da fase residual na zona vadosa (Fetter (1993)). No movimento descendente o LNAPL pode deslocar a água e 0 ar da zona vadosa e se acumular na zona capilar (Fase Livre). Esta zona pode desaparecer completamente migrando segundo o declive do nível de água, ficando o hidrocarboneto preso na zona saturada que é denominada fase dissolvida e consequentemente 0 aumento dos contaminantes e a adsorção dos mesmos pela matéria orgânica designa-se como fase adsorvida.

O transporte na zona saturada basicamente depende da velocidade das águas subterrâneas, taxa de migração, dispersão, difusão, volatização e sorção. Enquanto a transformação dos contaminantes dissolvidos é afetada pelos processos químicos como a ionização, solubilidade $e$ hidrólise, oxidação/redução, como também por processos biológicos de bioacumulação e biodegradação.

Moreira and Dourado (2007) explicam que a biodegradação contínua dos hidrocarbonetos aromáticos presentes no meio possivelmente resultou em elevadas concentrações de ácidos orgânicos, produzindo dissolução mineral, com aumento da porosidade e elevação nos teores de sólidos totais dissolvidos. Explicando assim que a existência de neoformação mineral nas diferentes fases de contaminação continua durante o tempo em que este permanecer no meio.

\section{Modelo Direto}

Para realizar a análise proposta e identificar as principais mudanças de comportamento de ondas eletromagnéticas diante as mudanças físicas provocadas pelos contaminantes, foi utilizado o software matGPRMatlab. Para isso, elaboraram-se três modelos diretos:
1. Modelo 1 - Antes da contaminação: Tanque subterrâneo de armazenamento de combustível de um posto de abastecimento.

2. Modelo 2 - Contaminação do solo: Vazamento de combustível demarcando as fases de contaminação.

3. Modelo 3 - Remediação do solo: Neoformação de minerais durante a contaminação, alterando o material arenoso saturado.

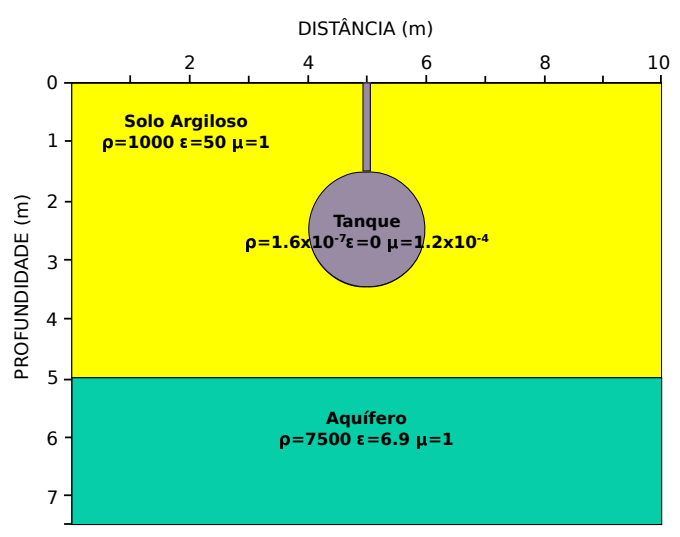

Figure 1: Modelo sintético antes da contaminação do solo com tanque de aço carbono subterrâneo e interface das camadas geológicas de material argiloso e arenoso saturado.

A geometria utilizada para a elaboração dos modelos foi de: $10 \mathrm{~m}$ de longitude e $7.5 \mathrm{~m}$ de profundidade, ver tabela 1. Esta foi dividida em duas camadas geológicas principais, a primeira contendo solo argiloso que vai de Om a 5m (Zona Vadosa) e a segunda constituída de material arenoso saturado de $5 \mathrm{~m}$ a $7.5 \mathrm{~m}$ (Zona Saturada), o tanque de aço tem um diametro de $2 \mathrm{~m}$, enterrado a uma profundidade de $1.5 \mathrm{~m}$ (ver figura 1). Também considerase a existência das fases de contaminação, a residual de $3.1 \mathrm{~m}$ a $5 \mathrm{~m}$, a fase livre de $5 \mathrm{~m}$ a $7.5 \mathrm{~m}$ (ver figura 2) e após a remediação de solo, gerou-se a neoformação de minerais (alteração do material arenoso saturado) e considerou-se essa camada como "solo recuperado" com espessura de aproximadamente $1 \mathrm{~m}$ que va desde $5 \mathrm{~m}$ a $6 \mathrm{~m}$ como se constata na figura 3.

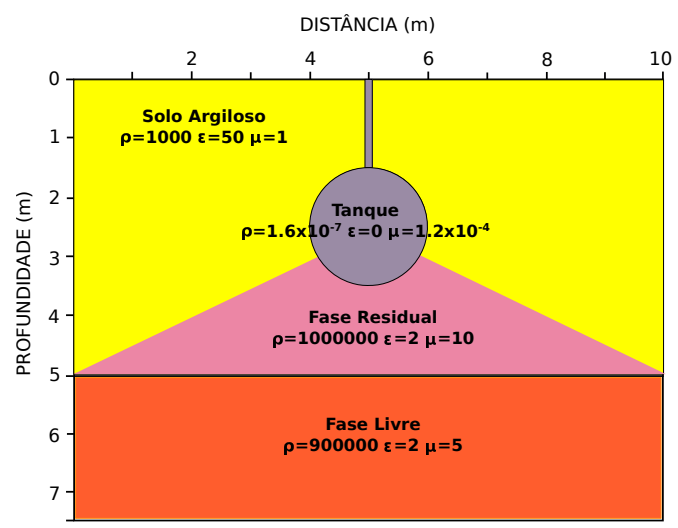

Figure 2: Modelo sintético com as fases de contaminação residual e livre. 


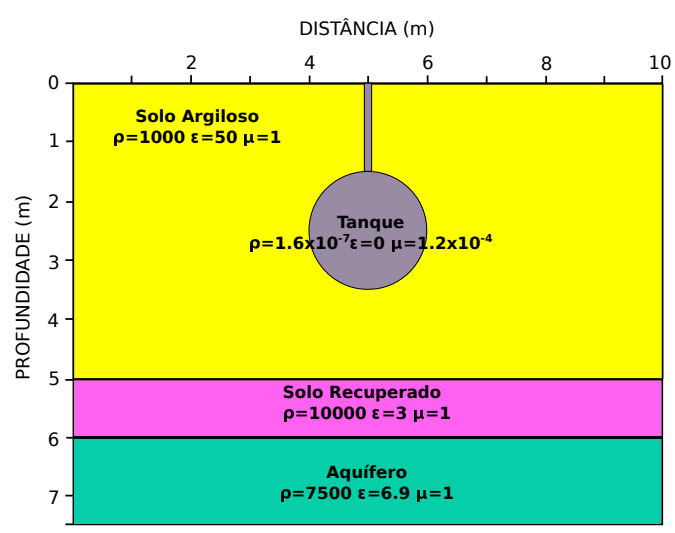

Figure 3: Modelo sintético de solo remediado e neoformação de minerais no material arenoso saturado.

\begin{tabular}{ccc}
\hline Material & Distância $(\mathrm{m})$ & Profundidade $(\mathrm{m})$ \\
\hline Solo Argiloso & $0 \mathrm{a} 10$ & $0 \mathrm{a} 5$ \\
Areia Saturada & 0 a 10 & $5 \mathrm{a} 7.5$ \\
Tanque & $4 \mathrm{a} 6$ & $1.5 \mathrm{a} 3.5$ \\
BTEX (Fase Residual) & 0 a 10 & $3.1 \mathrm{a} 5$ \\
BTEX (Fase Livre) & 0 a 10 & $5 \mathrm{a} 7.5$ \\
Recuperação do solo & $0 \mathrm{a} 10$ & $5 \mathrm{a} 6$ \\
\hline
\end{tabular}

Table 1: Valores utilizados na modelagem para os diferentes materiais

Baseando-se em Dehaini (2001) os parâmetros elétricos como resistividade, constante dielétrica relativa e a permeabilidade magnética, utilizados na contrução dos modelos tem valores dispostos na tabela 2. Desde um ponto de vista geofísico, as plumas de contaminação de hidrocarbonetos, possui caraterísticas elétricas resistivas (Atekwana et al. (2000)) o qual foi designado ao modelo 2.

\section{Simulação do modelo Direto}

Uma vez atribuidos os valores aos modelos diretos, obteve-se as seguintes radargramas:

Modelo 1: Antes do vazamento Na figura 4 observam-se duas hipérboles, uma acentuada a um tempo de 70ns e a outra a 130ns, sendo estas o topo e base do tanque de aço carbono respectivamente. Assim mesmo, na delimitação entre o material argiloso e o material arenoso, tem-se demarcado um refletor a um tempo de $240 \mathrm{~ns}$.

Modelo 2: Contaminação do solo - aquífero Observouse no radargrama a presença de uma zona sombra em lugar do refletor a 240ns, atenuando quase totalmente a

\begin{tabular}{cccc}
\hline Material & $\rho$ & $\varepsilon$ & $\mu$ \\
\hline Solo Argiloso & 1000 & 50 & 1 \\
Areia Saturada (Aquífero) & 7500 & 19 & 1 \\
Aço Carbono (Tanque) & $1.6 \times 10^{-7}$ & 0 & $1.2 \times 10^{-4}$ \\
BTEX (Fase Residual) & 1000000 & 2 & 10 \\
BTEX (Fase Livre) & 900000 & 2 & 5 \\
Recuperação do solo & 10000 & 3 & 1 \\
\hline
\end{tabular}

Table 2: Valores de resistividade [ $\rho$ (ohm.m)], constante dielétrica $(\varepsilon)$ e permeabilidade magnética $[\mu(\mathrm{T} . \mathrm{m} / \mathrm{A})] \mathrm{de}$ cada material.

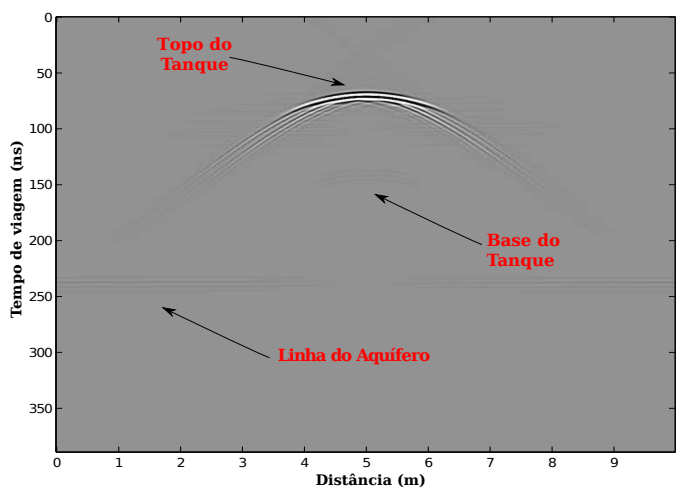

Figure 4: Radargrama antes da contaminação com antena de $250 \mathrm{MHz}$

interface entre o material argiloso e o aquífero. ver figura 5 .

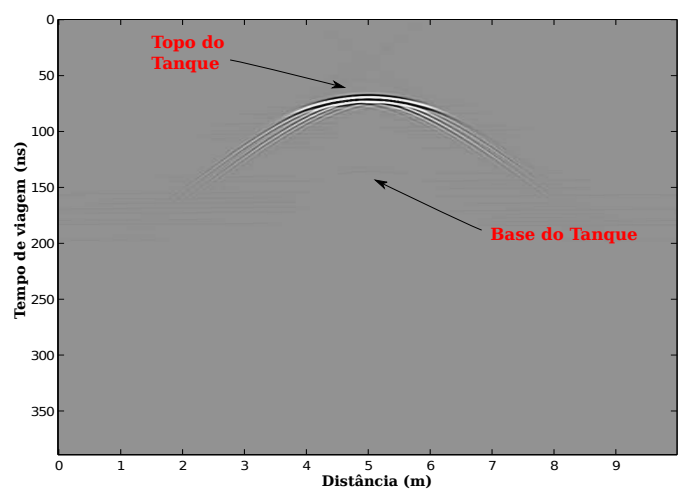

Figure 5: Radargrama após a contaminação e identificação da zona de sombra.

Modelo 3 - Remediação do solo: Neste radargrama observa-se o retorno do refletor entre um tempo de 240ns e 250ns, correspondente a interface entre a argila e o aquífero recuperado com a neoformação dos minerais. Ver figura 6

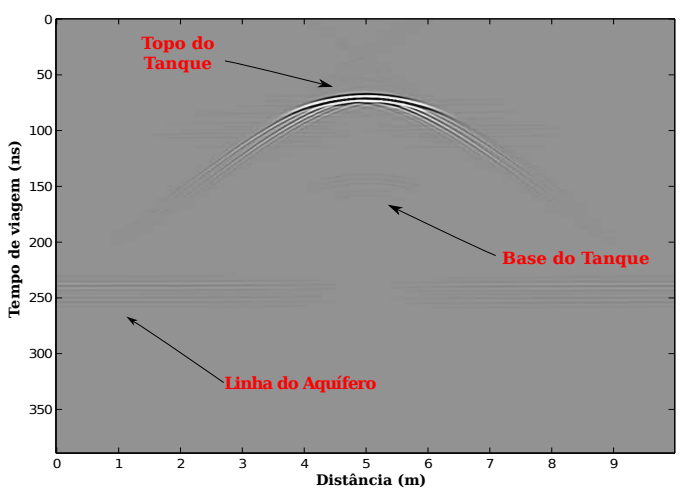

Figure 6: Radargrama após a remediação do solo e interface do material arenoso saturado com neoformação dos minerais 


\section{Analise do comportamento da onda EM}

Segundo Chelmus (2016), a validação da interpretação dos radargramas usando a modelagem e simulação de propagação da onda eletromagnética de objetos enterrados e estratigrafias, pode ser utilizada na ausência de dados adquiridos in situ, a fim de entender melhor a propagação da onda em ambientes complexos. Neste trabalho, pretendeu-se verificar se após a remediação do solo, o ambiente geológico recupera as propriedades físicas como se este não tivesse sido contaminado antes.

Muitas vezes, a falta de monitoramente das propriedades físicas e análise do comportamento da onda EM do solo antes da implementação do posto, facilitaria de forma rápida o monitoramento após uma possível contaminação, tal como a delimitação, distribução, direção do fluxo dos contaminantes, dessa forma, evitaria-se a perfuração de excessivos poços de monitoramento.

\section{Resultados}

Foram realizados testes con antenas de $100 \mathrm{MHz}$ e $250 \mathrm{MHz}$ (figura 7) e quem proporcionou melhores resultados foi a de $250 \mathrm{MHz}$ provavelmente devido ao fato desta apresentar melhor resolução do que a de $100 \mathrm{MHz}$. Neste caso há a necessidade de avaliar a relação resoluçãoxprofundidade, pois as seções produzidas pela antena de $100 \mathrm{MHz}$ apresentam zona de sombra de forma mais sutil que a antena de $250 \mathrm{MHz}$, ver figuras $8 \mathrm{a}$ e $8 \mathrm{~b}$.

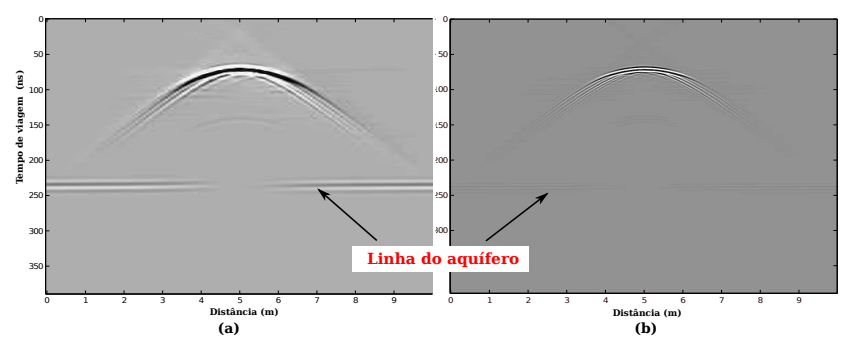

Figure 7: Comparação do modelo antes da contaminação a) antena de $100 \mathrm{MHz}$ e b) antena de $250 \mathrm{MHZ}$

Notou-se também a ausência de reflexão logo abaixo do tanque de aço, denominada de zona de sombra ou shadow zone, e corresponde a atenuação da amplitude do sinal provocada pela alta condutividade do meio aparentemente devido aos produtos e subproductos da biodegradação de hidrocarbonetos. Esta zona de sombra atenuou-se quase por completo a uns 240ns, como mostra a figura 8, este fato é explicado por Moreira and Dourado (2007), Dehaini (2001) Rodrigues and Porsani (2006).

Pode-se entender a razão entre 0 aumento da condutividade elétrica e a evidência da zona de sombra, posto que, a medida que a remediação do solo se leve a cabo ao longo dos anos, a disperção dos contaminantes iônicos aumente e a degradação dos hidrocarbonetos por ação da bactérias incrementes nas diferentes fases de contaminação, isso reflete diretamente no parâmetro físico da condutividade, realçando ou atenuando o refletor (ver figura 9), permitindo assim um monitoramente ao longo da remediação.

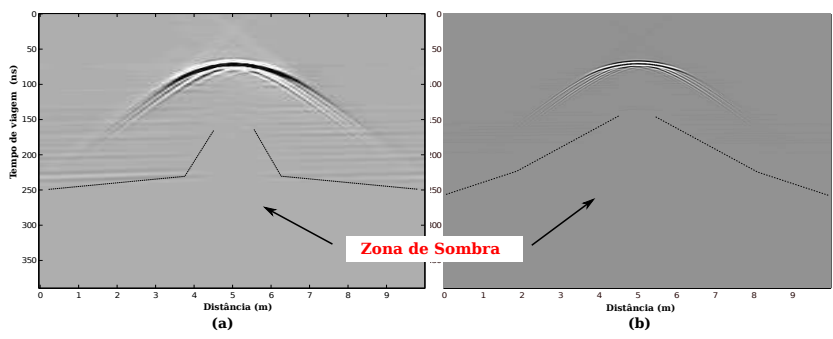

Figure 8: Comparação do modelo com as fases de contaminação a) antena de $100 \mathrm{MHz}$ e b) antena de 250MHZ. Atenuação da onda EM devido à biodegradação do hidrocarboneto

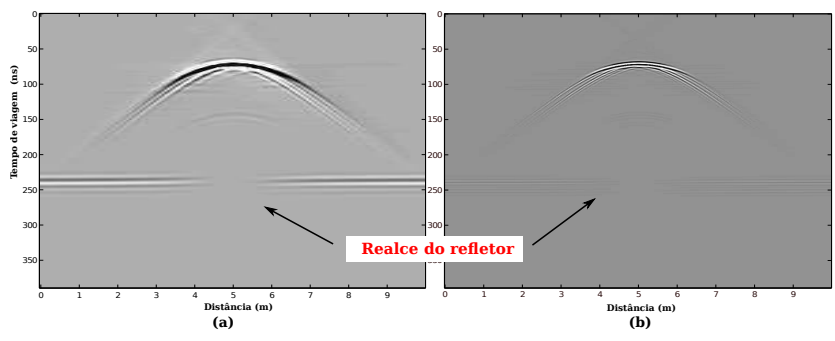

Figure 9: Comparação do modelo com solo remediado e realce do refletor a) antena de $100 \mathrm{MHz}$ e b) antena de $250 \mathrm{MHZ}$

\section{Conclusões}

Neste trabalho teve-se como objetivo a análise de monitoramento antes de um possível vazamento, avaliando os diferentes ambientes geológicos sem a presença do hidrocarboneto. Este comportamento foi visualizado na figura 4. Para o segundo modelo, segundo Dehaini (2001), e Moreira and Dourado (2007), a zona de sombra no radargrama é causada pelos componentes do BTEX (figura 5) e após a remediação da pluma de contaminação, ao longo de 5 a 10 anos, a zona de sombra diminui ressaltando o material geológico original, pode-se dizer que zonas insentas de contaminação apresentam a formação do refletor. Isso foi constatado no modelo 3 na figura 6 , visualizando o refletor entre o solo argiloso e o material arenoso saturado com neoformação de novos minerais, causado também pelos agente químicos, biológicos e fisicos agindo no solo. O refletor mostrado a 240ns assemelha-se ao modelo original. Também, fez-se o experimento com 2 diferentes antenas (100 e $250 \mathrm{MHz}$ ) e a diferentes profundidades, obteve-se em ambos os modelos a zona de sombra, ver figura 5 e 8 , sendo mais evidente na antena de $250 \mathrm{MHz}$ por conta da resolução/produndidade . Com isso, conseguiuse avaliar a aplicabilidade do GPR ante situações de monitoramento conforme o exemplo de dado real de Dehaini (2001) e além disso avaliar o solo antes de este ser contaminado e constatar que além do controle pontual dos poços de monitoramento do posto, pode-se monitorar a extensão lateral e vertical de forma gradual e rápida, auxiliando assim no controle da qualidade da água. Tem-se também como preocupação a profundidade dos poços de monitoramento, que muitas vezes é inferior à profundidade das plumas dos contaminantes, dificultando assim a delimitação vertical do poluente. Desta forma, 
com o monitoramento por GPR, e a escolha da antena adequada, tornaria-se a melhor o controle do mesmo.

\section{References}

Atekwana, E. A., W. A. Sauck, and D. D. Werkema, 2000, Investigations of geoelectrical signatures at a hydrocarbon contaminated site: Journal of Applied Geophysics, 44, 167 - 180.

Chelmus, A., 2016, Validation of ground penetrating radar data interpretation using an eletromagnetic wave propagation simulator: Romanian Reports in Physics, 68, 1584-1588.

de Castro, D. L., and R. M. G. C. Branco, 2003, 4-d ground penetrating radar monitoring of a hydrocarbon leakage site in fortaleza (brazil) during its remediation process: a case history: Journal of Applied Geophysics, 54, 127 144.

Dehaini, J., 2001, Detecção da pluma de contaminação de hidrocarbonetos em subsuperfície pelo método de radar de penetração: Dissertação de mestrado, Universidade de São Paulo.

Fetter, C., 1993, Contaminant hydrogeology: Contaminant hydrogeology.

Lima, R. M. S., 2010, Avaliação de risco à saúde humanadecorrentes dacontaminação da Água subterrânea por btex provenientes de postos de revenda de combustíveis em manaus: Dissertação de mestrado, Universidade Federal do Amazonas.

Mello, J. M. M., 2007, Biodegradação dos compostos btex em um reator com biofilme: Dissertação de mestrado, Universidade Federal de Santa Catarina.

Moreira, C., and J. C. Dourado, 2007, Monitoramento da atenuação natural de pluma de contaminação élo método de radar de penetração no solo (GPR): Revista Brasileira de Geofísica, 25, 389 - 398.

Penner, G. C., 2005, Estudo de caso da contaminação do solo e da água subterrânea por descarte de resíduos vinculados a óleos lubrificantes: Dissertação de mestrado, Universidade de São Paulo.

Prommer, H., G. Davis, and D. Barry, 1999, Pht3d-a three-dimensional biogeochemical transport model for modelling natural and enhanced remediation. contaminated site remediation: Challenges posed by urban and industrial contaminants: 351-358.

Rodrigues, S. I., and J. L. Porsani, 2006, Utilização do GPR para caracterizar tambores plásticos enterrados no sítio controlado de Geofísica rasa do IAG/USP: Revista Brasileira de GeofÃsica, 24, 157 - 168.

Tiburtius, E. R. L., P. Peralta-Zamora, and E. S. Leal, 2004, Contaminação de águas por BTXs e processos utilizados na remediação de sítios contaminados: Química Nova, 27, 441 - 446. 